\title{
Host Dendritic Cell Vaccine-001 MSSM/BIIR
}

National Cancer Institute

\section{Source}

National Cancer Institute. Host Dendritic Cell Vaccine-001 MSSMIBIIR. NCI Thesaurus.

Code $C 84845$.

A dendritic cell (DC) vaccine containing ex vivo expanded autologous DCs obtained from a patient with leukemia with potential immunostimulating activity. Upon reintroduction into the host, the host dendritic cell vaccine-001 MSSM/BIIR may stimulate the immune system to mount a leukemia-specific cytotoxic T lymphocyte (CTL) response. 